\title{
Nanocontrol of single dense energetic electron sheet in a chirped pulse with critical relativistic intensity
}

\author{
Luling Jin, ${ }^{1,2}$ Meng Wen, ${ }^{1, *}$ and Baifei Shen ${ }^{3}$ \\ ${ }^{1}$ Institute of Photonics \& Photon-Technology, Northwest University, Xi'an 710069, China \\ ${ }^{2}$ Department of Physics, Northwest University, Xi'an 710069, China \\ ${ }^{3}$ Shanghai Institute of Optics and Fine Mechanics, Chinese Academy of Sciences, Shanghai 201800, China
}

(Received 31 January 2013; published 6 May 2013)

\begin{abstract}
We investigate the effect of laser frequency chirp on the generation of a dense energetic electron sheet. The direct acceleration regime of laser driven dense electron sheet requires high laser intensity to function efficiently. We demonstrate this requirement can be relaxed by applying chirped lasers. The acceleration becomes more efficient while driven by a pulse with a proper linear chirp, leading to a dense energetic electron sheet in nanoscale, and therefore requires lower laser intensity.
\end{abstract}

\section{INTRODUCTION}

The rapid developments in laser technology have allowed the production of ultrashort and ultraintense lasers [1]. The interaction of matter with such fields is capable of producing high energy electrons, ions, as well as photons. In particular, the wave reflected at a laser driven relativistic electron bunch can be a very potential compact $\mathrm{x}$-ray source [2-4], which attracts more and more attention due to its application on the detection of matter [5]. Electron bunches with $\mathrm{GeV}$ energy can be generated by bubble formation in plasma [6-10]. This efficient acceleration has been exploited to produce incoherent multi-keV xray pulses [2]. A proposal to generate bright $\mathrm{x}$-ray of coherent Thomson scattering on a dense energetic electron sheet (DEES) is to reflect counterpropagating photons off such a flying mirror with frequency upshift of $4 \gamma_{x}^{2}$, where $\gamma_{x}=1 / \sqrt{1-\beta_{x}^{2}}$ is the longitudinal relativistic factor of a DEES and $\beta_{x}=v_{x} / c$ is the corresponding velocity [4]. Except for the application of the DEES on the coherent Thomson backscattering, the nanocontrolled DEES is also capable of generating giant half-cycle attosecond pulses [11]. This kind of relativistic DEES is attainable with an ultrathin foil irradiated by an ultraintense laser $[12,13]$.

It is shown that there is a condition to generate a well confined DEES in the laser foil interaction [13], which is the normalized field amplitude $E_{L 0}$ must be much larger than the longitudinal electrostatic field due to the charge separation between electrons and ions $E_{x}$. There are two severe requirements to fulfill this condition. First, an ultrathin foil is needed to reduce the requirement on the laser intensity. The potential target can be the diamondlike

\footnotetext{
*mengwen@nwu.edu.cn
}

Published by the American Physical Society under the terms of the Creative Commons Attribution 3.0 License. Further distribution of this work must maintain attribution to the author(s) and the published article's title, journal citation, and DOI. carbon foil [12] or nanomeshed graphene [14]. Second, even with a nanofoil target, a very sharp rising edge and high intensity are also needed to avoid the heating and expansion effect of the electron sheet before the efficient acceleration [12]. As a fact, this kind of single dense electron bunch could previously only be fulfilled by using nonadiabatic [13] or quasisingle cycle intense pulse [11]. It is worth mentioning that a DEES with density $n_{e} \gg$ $10^{13} \gamma_{x}^{4} \mathrm{~cm}^{-3}$ is needed in this potential compact coherent $\mathrm{x}$-ray generation regime [15]. In this way, the DEES need to be controlled in nanoscale to keep its high density, while it is from a nanofoil with solid density, e.g., a nanomeshed graphene target.

In many laser particle acceleration regimes such as those mentioned above, laser fields with a constant frequency were adopted, thus appearing several limits on bunch confinement [16], net energy gain $[17,18]$, energy spread [19], etc. If the instantaneous frequency of the laser pulse varies with time, the limits mentioned can be overcome in some degree. The interaction of laser with particles can show qualitatively different results if the frequency changes along the pulse length. Such pulses are used to generate intense femtosecond pulses in solid-state laser systems, which is called chirped pulse amplification [20]. With current technology, the chirp of an intense pulse can be adjusted with great flexibility [21,22]. The normalized spectral bandwidth of a few-cycle laser pulse can extend to a few tens of percents [23]. Many works have been done to study the chirp effect on laser matter interactions, such as high-order harmonic generation [24], Raman instabilities [25], and hot electron production [26]. Specifically, several schemes of chirped pulse driven acceleration in vacuum and plasma have been proposed [27-35], in which the chirped pulse shows great potential on the acceleration efficiency, i.e., resulting in much more energetic particles. As for the DEES, the Coulomb expansion also needs to be considered in the laser foil acceleration [16], which would lead to wider energy spectrum and lower density. In this 
topic, circularly polarized laser pulses are more favorable to inhibit the Coulomb expansion than linearly polarized pulses [19], and could generate electron sheets with lower energy spread.

In this paper, we are interested in whether the chirped pulse is beneficial to the confinement of the DEES, as well as the inhibition of the energy spread. The feasibility of generating a DEES from illuminating a nanomeshed graphene foil with a properly chirped laser pulse is demonstrated. In order to show the relaxation of the requirement on laser intensity by introducing a proper chirp, we use a chirped pulse with its intensity as low as the critical relativistic intensity $\left(\sim 10^{18} \mathrm{~W} / \mathrm{cm}^{2}\right)$. The organization of this paper is as follows: In Sec. II, the chirp effect on electron acceleration is performed so as to find a proper chirp parameter. In Sec. III, the confinement of a DEES surfing in a chirped laser pulse is investigated numerically. The verification with particle-in-cell (PIC) simulations is provided in Sec. IV. Summary and conclusions are presented in Sec. V.

\section{CHIRP EFFECT ON ELECTRON ACCELERATION}

In this investigation, electrons from an ultrathin foil are accelerated by a linearly chirped intense laser pulse. For convenience, we shall use the normalized quantities, i.e., time and space coordinates are normalized according to $t^{\prime}=\omega_{0} t$ and $x^{\prime}=k_{0} x$, velocity $\beta=v / c$, momentum $p^{\prime}=p / m c$, density $n^{\prime}=n / n_{c}$, fields $E^{\prime}=e E /\left(m c \omega_{0}\right)$, $B^{\prime}=e B /\left(m c \omega_{0}\right)$, and vector potential $a=e A /\left(m c^{2}\right)$, where $e$ and $m$ are the charge and the mass of the electron, $c$ is the speed of light in vacuum, $\omega_{0}$ and $k_{0}$ are the unchirped (central) laser frequency and the wave number (corresponding a laser period $\tau_{0}$ and a wavelength $\lambda_{0}$ ), and $n_{c}=m \omega_{0}^{2} /\left(4 \pi e^{2}\right)$ is the critical electron density. In the following, these normalized quantities are used dropping the prime.

We consider a linearly chirped pulse propagates in vacuum along normal direction $x$, which is linearly polarized in transverse direction $y$. In the paraxial approximation, the electric field component of a laser pulse with a cosine square envelope is written as

$$
E_{L y}=E_{L 0} \cos ^{2}\left(\frac{\pi \tau}{2 \sigma_{T}}\right) \cos \Phi
$$

where $E_{L 0}$ is the constant amplitude, $\tau=t-x-\bar{\tau}$ is the retarded time, $\bar{\tau}=\sigma_{T}$ is a shift of $\tau$ to ensure a particle from rest at $x=0, \sigma_{T}$ is the laser pulse duration at fullwidth half-maximum (FWHM), $\Phi=\tau+b \tau^{2}+\phi_{0}$ is the absolute oscillating phase, $b$ is the chirp parameter, and $\phi_{0}$ is the initial phase. The laser fields satisfy the dispersion relation $\omega=c k$ and $B_{L z}(\tau)=E_{L y}(\tau)$. The chirp form is controlled by the chirp parameter $b$. The instantaneous frequency is then of the form $\omega(\tau)=\omega_{0}(1+b \tau)$. When $b=0$, the pulse is chirp free. Figure 1(a) shows an un-
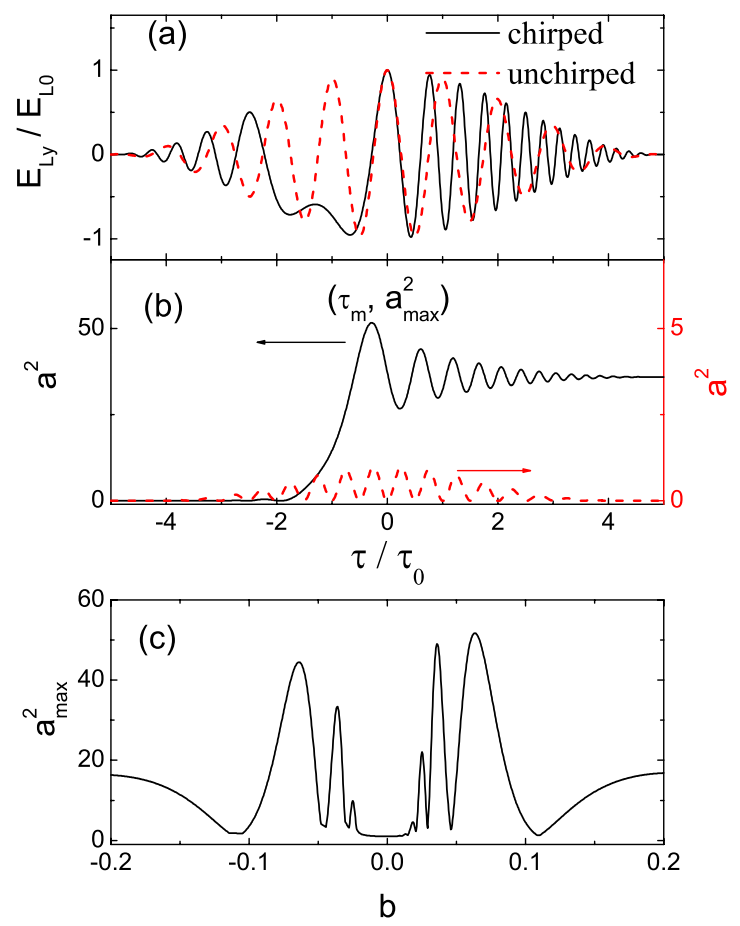

FIG. 1. (a) The normalized electric fields of plane wave pulses as Eq. (1) with $\phi_{0}=0$ and $\sigma_{T}=5 \lambda_{0}$. The chirp parameter for the chirped pulse is $b=0.0635$. (b) The corresponding parameters $a^{2}$ depending on $\tau$ of chirped and unchirped pulses. Here $E_{L 0}=m c \omega_{0} / e$. The solid (black) curves represent a chirped pulse and the dashed (red) curves represent an unchirped pulse. The maximum value of $a^{2}$ in (b) is plotted as a function of chirp parameter $b$ in (c).

chirped (chirped) pulse as a function of $\tau$. Because of the plane wave symmetry, interaction of particles with the unchirped pulse in vacuum results in no net energy gain from laser, which is the so-called Lawson-Woodward theorem $[17,18]$. The chirp provides a pathway to enhance the asymmetry of the electron surfing in the wave pulse, resulting in energy gain.

What is particularly important to us is the confinement of the DEES while surfing in a chirped pulse. This effect will be investigated in the next section. To begin with, the chirp effect on the electron acceleration potential needs to be investigated to find a proper chirp parameter. In this case, an analytical treatment for a free electron motion in a electromagnetic field is applied [4]. The transverse momentum of electron sheet is dominated by the transverse laser field,

$$
p_{y} \sim \int_{-\infty}^{\tau} E_{L y} d \tau=a(\tau)
$$

where $a(\tau)$ is the normalized laser vector potential. Here we use the relation $\gamma \sim a^{2}(\tau)$ (see Sec. 2.2 in Ref. [4]) to define the acceleration effect.

The asymmetry of a chirped laser pulse, as shown by the solid (black) curve in Fig. 1(a), leads to an asymmetric 
acceleration. Although a linearly chirp parameter is adopted in the pulse, the electric oscillation increases in frequency after a decreasing stage, instead of varying monotonously. A period of low frequency integration of $E_{L y}$ in Eq. (2) leads to a large value of $a^{2}(\tau)$. The dependence of $a^{2}(\tau)$ on $\tau$ is presented in Fig. 1(b). It is found there is a main accelerating stage (MAS), which begins at the time $\tau_{s}=-1.9 \tau_{0}$ when slowly varying of $E_{L y}$ appears, and ends at $\tau_{m}=-0.3 \tau_{0}$ when $a^{2}(\tau)$ reaches a maximum value $a_{\max }^{2}$ as marked in Fig. 1(b). Here $a_{\max }=$ $\int_{-\infty}^{\tau_{m}} E_{L y} d \tau$. The duration $\tau<\tau_{s}$ represents the length of a weak oscillation stage before the MAS, during which the electrons gain much lower energy than in the MAS.

The most appreciable energy gain of an electron comes from the interaction with the laser fields in the MAS $\left[\tau_{s}, \tau_{m}\right]$. After that, $a^{2}(\tau)$ reduces about $30.5 \%$ and oscillates around the constant value until the electron leaves the laser fields. The parameter $a_{\max }^{2}$ is plotted in Fig. 1(c), as a function of the chirp parameter $b$. The reason we use the integration over part of the laser pulse $\left[-\infty, \tau_{m}\right]$ is that accelerated electrons can be extracted by applying a thick reflect foil at a proper position. The thick reflect foil with large normalized area density $\left(n_{r} d_{r} \gg a_{\max }\right)$ is used to reflect the laser pulse, but let the electrons pass through [36]. Otherwise, if the electrons still surf in the laser pulse after the MAS, the laser pulse may increase the expansion of electron sheet in phase space, and the DEES will not be obtained. The expansion effect will be discussed in the next section.

The total integration over the entire pulse $\int_{-\infty}^{\infty} E_{L y} d \tau$ leads to the same value with chirp parameters of different sign but same absolute value $|b|$ [35]. However, the dependence of $a_{\max }^{2}$ on $b$ is asymmetrical, shown in Fig. 1(c). Pulses with positive chirp have larger $a_{\max }^{2}$ than negatively chirped pulses with same $|b|$. The flat low portion $\left(a_{\max }^{2} \approx 1\right)$ around $b=0$ corresponds to the LawsonWoodward theorem $[17,18]$. The maximum peak of the curve appears at $b=0.0635$. It means the electron acceleration in the chirped pulse with $b=0.0635$ has the strongest acceleration in the MAS. This optimized chirp parameter $b$ is codominated by the initial phase $\phi_{0}$ and the duration $\sigma_{0}$ mentioned in Eq. (1). A few-cycle laser pulse with the initial phase $\phi_{0}=0$ and the FWHM duration $\sigma_{s}=5 \lambda_{0}$ is applied in this paper.

\section{NANOCONTROL OF THE DEES IN PHASE SPACE}

Let us study the evolution of a DEES in a chirped laser pulse over a finite interaction region $\tau \in\left[-\sigma_{T}, \tau_{m}\right]$, during which the DEES is accelerated from rest to the maximum energy $\left(\gamma=\gamma_{m}\right)$. We investigate the dynamics in the electromagnetic field propagating in the normal direction. Different from Sec. II, interaction among the electrons in a DEES should be considered. In this case, the total electromagnetic fields $\mathbf{E}=\mathbf{E}_{L}+\mathbf{E}_{s}$ and $\mathbf{B}=\mathbf{B}_{L}+\mathbf{B}_{s}$ include the self-fields $\mathbf{E}_{s}$ and $\mathbf{B}_{s}$ of the DEES in addition to the external chirped laser field as Eq. (1). The self-fields consist of the longitudinal electrostatic field $E_{s x}$ and the transverse electromagnetic fields $E_{s y}$ and $B_{s z}$ due to the transverse electron currents. The electron sheet surfs in the fields, while the ions are taken as immobile particles. The ultrathin foil thickness and density are $d_{0}$ and $n_{0}$, respectively. The initial position $x_{0}$ of an electron sublayer in the ultrathin foil $0<x_{0}<d_{0}$ is taken as the Lagrangian coordinate. The self-fields, felt by the sublayer of initial position $x_{0}$ and current pushed out of the foil, are presented as

$$
\begin{gathered}
E_{s x}= \begin{cases}n_{0}\left(x-x_{0}\right) & (x \leq d) \\
n_{0}\left(d_{0}-x_{0}\right) & (x>d),\end{cases} \\
E_{s y}=\frac{n_{0} \beta_{y}}{2}\left[\frac{x_{0}}{1-\beta_{x}}+\frac{d_{0}-x_{0}}{1+\beta_{x}}\right], \\
B_{s z}=\frac{n_{0} \beta_{y}}{2}\left[\frac{x_{0}}{1-\beta_{x}}-\frac{d_{0}-x_{0}}{1+\beta_{x}}\right] .
\end{gathered}
$$

Equation (3) describes $E_{s x}$ increases linearly with $x$ while the sublayer is still inside the ion volume and becomes constant after leaving the ion layer. This corresponds to the attraction from the ion layer and the Coulomb expansion in the electron layer. In Eqs. (4) and (5), the first and second terms in the square brackets denote the radiation from the left and right electron currents of the considered layer with initial position $x_{0}$, respectively.

By considering the self-fields as well as the external laser fields, the dynamics of an electron sublayer are expressed as

$$
\begin{gathered}
\frac{d}{d t}\left(\gamma-p_{x}\right)=\left(1-\beta_{x}\right) E_{x}-\beta_{y}\left(E_{y}-B_{z}\right), \\
\frac{d p_{y}}{d t}=-\left(1-\beta_{x}\right) E_{y}-\beta_{x}\left(E_{y}-B_{z}\right),
\end{gathered}
$$

where $E_{x}=E_{s x}, E_{y}=E_{L y}+E_{s y}$, and $B_{z}=B_{L z}+B_{s z}$. By introducing $\kappa=\gamma-p_{x}$ and $\tau=t-x-\bar{\tau}$, the coupled equation can be written as

$$
\begin{gathered}
\frac{d \kappa}{d \tau}=E_{s x}-n_{0}\left(d_{0}-x_{0}\right) \frac{p_{y}^{2}}{1+p_{y}^{2}} . \\
\frac{d p_{y}}{d \tau}=-E_{L y}-\frac{n_{0} d_{0}}{2} \frac{p_{y}}{\kappa} .
\end{gathered}
$$

We perform classical calculations of the DEES dynamics to facilitate the investigation of confinement responsible for the chirp parameter. A fully ionized nanomeshed graphene [14] is used as the initial ultrathin foil of the density $n_{0}=30 n_{c}$ and the thickness $d_{0}=0.001 \lambda_{0}$. The DEES is divided into 100 sublayers, and the movement of each sublayer is assumed to obey Eqs. (8) and (9). The 
solutions $p_{y}(\tau)$ and $\kappa(\tau)$ can be calculated by numerically integrating this coupled equation over the duration $\left[-\sigma_{T}, \tau\right]$. With $\gamma^{2}=1+p_{x}^{2}+p_{y}^{2}$, one can obtain

$$
\begin{aligned}
& p_{x}(\tau)=\frac{1+p_{y}^{2}}{2 \kappa}-\frac{\kappa}{2}, \\
& \gamma(\tau)=\frac{1+p_{y}^{2}}{2 \kappa}+\frac{\kappa}{2}, \\
& x(\tau)=\int_{-\sigma_{T}}^{\tau} \frac{p_{x}}{\kappa} d \tau,
\end{aligned}
$$

in parametric form with time given $t(\tau)=\tau+x(\tau)+\sigma_{T}$. In this way, the evolution as well as the trajectories of sublayers of a DEES are performed below.

The energy evolution of different sublayers of a DEES along the spacial coordinate $x$ during a chirped pulse is plotted in Figs. 2(a) and 2(b), while Fig. 2(c) shows the corresponding time dependent variations of the phases $\Phi$ of $E_{L y}$ in Eq. (1). In these calculations, the laser amplitude is chosen to be $e E_{L 0} /\left(m c \omega_{0}\right)=1$, which corresponds to a critical relativistic intensity $\left(\sim 10^{18} \mathrm{~W} / \mathrm{cm}^{2}\right)$. When the laser pulse is properly chirped $(b=0.0635)$, the electrons
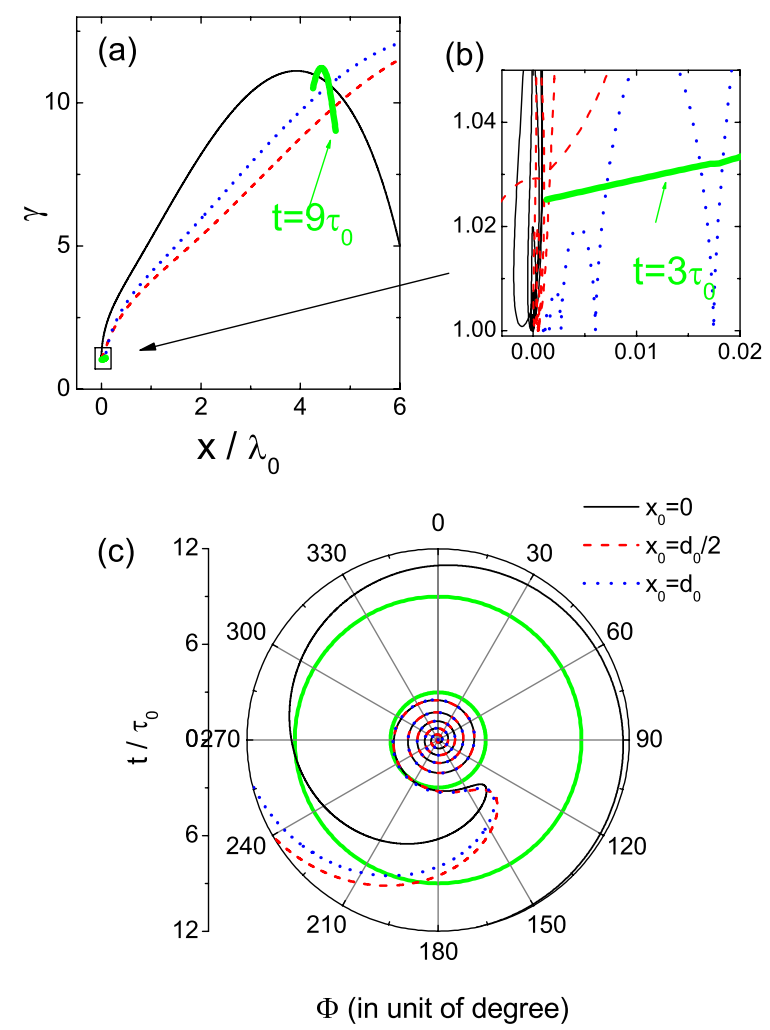

FIG. 2. (a) The spacial evolution of normalized energy of the initial tail $\left(x_{0}=0\right)$, middle $\left(x_{0}=d_{0} / 2\right)$, and front $\left(x_{0}=d_{0}\right)$ sublayers during the laser pulse. We zoom in part of (a) in (b). (c) The variation of $E_{L y}$ 's phase experienced by different sublayers. The chirped parameter is $b=0.0635$. can be pushed out from the ion layer and accelerated during the MAS as shown in Fig. 2(a). Sublayers are accelerated synchronously in the MAS in the duration $t \in\left[3 \tau_{0}, 9 \tau_{0}\right]$. This leads to a well confined DEES in phase space. The boundaries are marked by green solid curves in Figs. 2(a) and 2(b). Figure 2(b) shows weak oscillations of sublayers before the MAS in detail by zooming in a part of Fig. 2(a), during which most sublayers shake around their initial positions with ions. The $E_{s x}$ during this process is described by the upper part of Eq. (3), which leads to more compact layers [37]. After a weak oscillation stage, sublayers enter the MAS and become energetic synchronously and quickly. In contrast, at this intensity, the initial ultrathin foil cannot be accelerated by an unchirped laser pulse, and all sublayers shake around ion volume in the whole interacting process. An asynchronous acceleration may appear while the unchirped laser intensity is increased, e.g., $e E_{L 0} /\left(m c \omega_{0}\right)=10$. The accelerating effect will be performed in the next section by applying PIC simulations.

Figure 2(c) shows the trajectories of different sublayers driven by a chirped pulse in the polar coordinates. The curves are anticlockwise helixes before the MAS. Because of the chirp effect, the helix for different sublayers appears to bend back to the clockwise direction. The positive range $\left(90^{\circ} \rightarrow 0^{\circ} \rightarrow 270^{\circ}\right)$ and negative range $\left(90^{\circ} \rightarrow 180^{\circ} \rightarrow 270^{\circ}\right)$ represent the oscillated positive and negative field $E_{L y}$. According to Eq. (1), the positive and negative maxima appear at $\Phi=0^{\circ}$ and $180^{\circ}$, respectively. The sublayers roll synchronously in polar coordinates before the MAS $\left(t<3 \tau_{0}\right)$, as most sublayers stay around the initial ultrathin foil [see Figs. 2(b) and 2(c)]. Only the most front sublayers move forward weakly as shown by the (blue) dotted line in Fig. 2(b). As most of the sublayers still stay in the ion volume, the expansion of the electron sheet by Coulomb field is not evident. During the MAS, the range of $\Phi$ is a subset in the range $\left(90^{\circ} \rightarrow\right.$ $180^{\circ} \rightarrow 270^{\circ}$ ), where the cosine function has a negative value without sign changes [see Fig. 2(c)]. The relatively long time acceleration in the same direction during the MAS leads to a DEES. What is more, the transverse momentum $p_{y} \sim a$ during the MAS becomes much lager than before, thus appearing much larger $\beta_{y}$ in Eqs. (4) and (5), and larger $E_{s y}$ and $B_{s z}$ as results. This greatly contributes to the confinement control of the DEES [16]. The DEES in Fig. 2(a) shows of a thickness less than $\lambda_{0} / 2$ during the MAS.

\section{VERIFICATION BY PIC SIMULATION}

In order to make a comparison with the analytical model, 1D and 2D PIC simulations are presented using the code VORPAL [38]. In the 1D simulations, we compare the cases with unchirped and properly chirped pulse. A few-cycle laser pulse with a duration $\sigma_{T}=5 \tau_{0}$ and an initial phase $\phi_{0}=0$ is focused on a nanomeshed 
graphene target of area density $n_{0} d_{0}=0.03 n_{c} \lambda_{0}$. The chirp parameter is $b=0.0635$. For a directly comparison with the results in the section above, the ultrathin foil is initial located at $x=0$ and the simulation box starts at $x=-2 \lambda_{0}$, with full length of $15 \lambda_{0}$. The resolution is 10000 cells $/ \lambda_{0}$ with 20 particles per cell.

Figures 3(a) and 3(c) show the electron distributions in phase space at $t=9 \tau_{0}$ for the chirp-free and chirped case, respectively. The corresponding energy spectra are plotted in Figs. 3(b) and 3(d). In the chirp-free case, a laser amplitude of $e E_{L 0} /\left(m c \omega_{0}\right)=10$ is applied to ensure the electrons can be pushed out of the initial ultrathin foil and accelerated. This intensity corresponds to an intensity of $I=1.35 \times 10^{20} \mathrm{~W} / \mathrm{cm}^{2}$ for $\lambda_{0}=1 \mu \mathrm{m}$. As seen in Fig. 3(a), all the electrons are accelerated, but disperse to several lase cycles in phase space. Electrons in each cycle have a corresponding peak in spectrum as shown in Fig. 3(b). This periodically modulated electron distribution
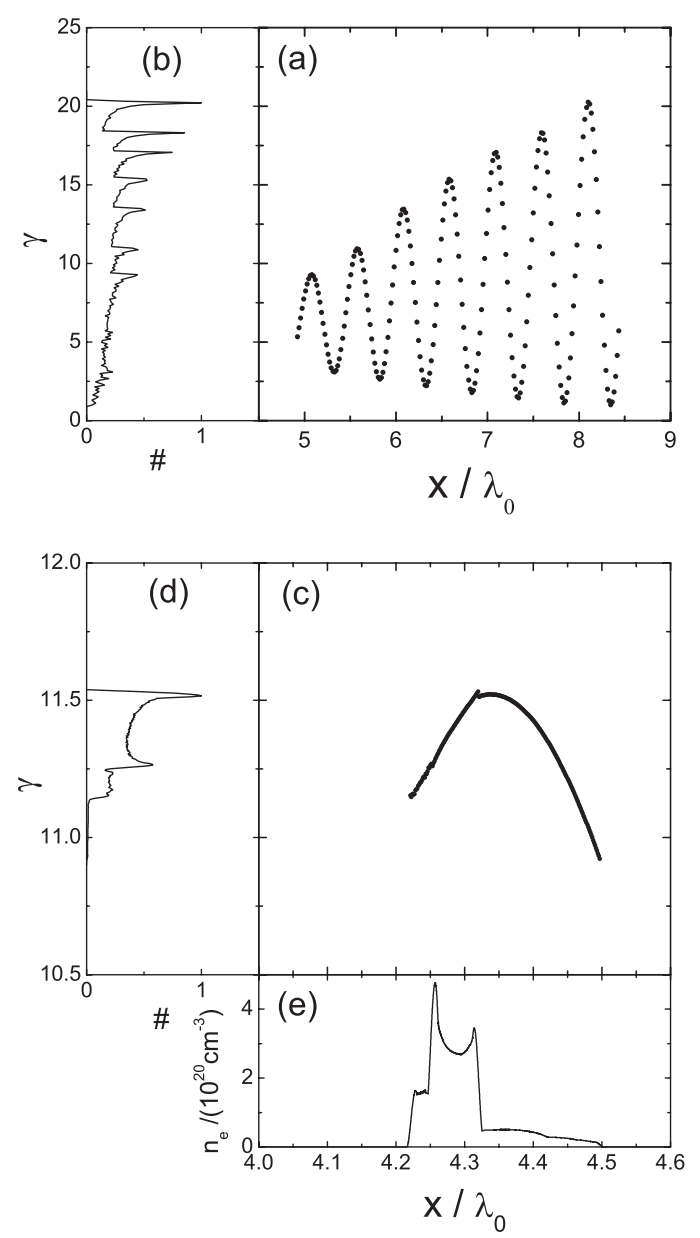

FIG. 3. Electron sheet distribution in phase space at $\tau=\tau_{m}$ for cases with unchirped (a) and chirped (c) pulses. (b), (d) and (e) are the corresponding energy spectra and density distributions of (a) and (c). The coordinate "\#" is the normalized counted number. The longitudinal coordinate of (e) is labeled as electron density. forms a "fingerprint" of laser field, which can be used to retrieve the waveform of laser field [39]. However, it cannot be used as a DEES for x-ray generation, due to its strong expansion. We also performed a simulation driven by a chirped-free pulse with $e E_{L 0} /\left(m c \omega_{0}\right)=1$, which is 100 times lower in intensity. In that case, the electrons cannot be accelerated but heated and shake around the initial ultrathin foil, which is similar with the case of $t<3 \tau_{0}$ of Fig. 2 .

The acceleration efficiency driven by the properly chirped pulse is much higher than the chirp-free case. We introduce a chirped pulse with critical relativistic intensity $I=1.35 \times 10^{18} \mathrm{~W} / \mathrm{cm}^{2}$ for $\lambda_{0}=1 \mu \mathrm{m}$, while the amplitude is $e E_{L 0} /\left(m c \omega_{0}\right)=1$. Figure 3(c) shows the energy of electrons can be $\gamma>11$ at $t=9 \tau_{0}$, which well reproduces the phase space distribution of electrons shown in Fig. 2(a). The electron energy is lower than that in Fig. 3(a), but the laser intensity used here is 100 times lower. The spectrum shows a narrow energy spread of $2.4 \%$ in Fig. 3(d). Meanwhile, the density keeps higher than $10^{20} \mathrm{~cm}^{-3}$ and the thickness is confined around $0.1 \lambda_{0}$, as seen in Fig. 3(e). It implies that a chirped laser pulse can drive a nanocontrolled DEES efficiently, even when a critical relativistically intense laser pulse is applied.

The 2D PIC simulation for a DEES driven by a chirped pulse is also carried out to see the effect of higher dimensions. The simulation box is $\left(-\lambda_{0} \sim 11 \lambda_{0}\right)_{x} \times\left(-10 \lambda_{0} \sim\right.$ $\left.10 \lambda_{0}\right)_{y}$ with the resolution of $\left(\lambda_{0} / 10000\right)_{x} \times\left(\lambda_{0} / 20\right)_{y}$ and 20 particles per cell. A chirped laser pulse is focused onto the left boundary, which has the same parameter as the 1D case and transverse super Gaussian envelope of the function $f=\exp \left[-y^{4} /\left(4 \lambda_{0}\right)^{4}\right]$. The $n_{0} d_{0}=0.03 n_{c} \lambda_{0}$

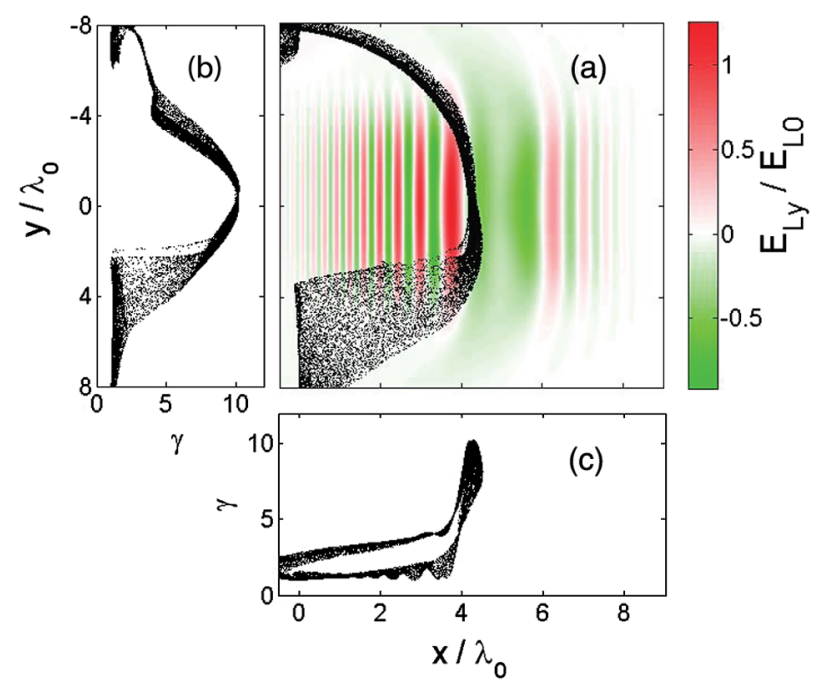

FIG. 4. The distribution of DEES driven by chirped pulse in $x-y$ space (a) and phase space [(b) and (c)] at $t=8 \tau_{0}$. The black dots (clouds) represent the simulation particles and the colored background in (a) represents the laser field. 
ultrathin foil is initially located at $x=0$ with a width $(-9 \mu \mathrm{m}-9 \mu \mathrm{m})_{y}$. Figure 4 shows the electron sheets distributions in $x-y$ space and phase spaces at $t=8 \tau_{0}$. Because of the 2D effect, particles are pushed aside from the pulse center, so that the MAS here is shorter than that in 1D case. The colored background in Fig. 4(a) represent the chirped laser pulse, which is slightly defocused while it propagates in the simulation box. Under the asymmetrically driven by the chirped pulse, the DEES becomes asymmetric along $y$ coordinate and its center moves downward to $y=-1.5 \lambda_{0}$, as seen in Figs. 4(a) and 4(b). However, the main features of the nanocontrolled DEES is the same as the $1 \mathrm{D}$ case. Comparing the cases of different dimension scale, Fig. 3(c) is a small part of Fig. 4(c), as 1D simulation only describe the condition of the center of the DEES. Different thicknesses of the DEES appear at different transverse positions as shown in Fig. 4(a), e.g. $\left.d \approx 0.1 \lambda_{0}\right|_{y=-1.5 \lambda_{0}},\left.\quad d \approx 0.24 \lambda_{0}\right|_{y=0.5 \lambda_{0}}$ and $d \approx$ $\left.0.17 \lambda_{0}\right|_{y=-3.5 \lambda_{0}}$. The nanocontrolled flattop DEES has a width of about $4 \lambda_{0}$ in the simulation, which can be enlarged by using laser pulse with wider waist. To realize the nanocontrolled DEES driven by a chirped laser pulse, a second thick and dense reflect foil can be set at $x_{r}=4 \lambda_{0}$ behind the initial ultrathin foil [36], where the MAS reaches the end under our simulation parameters.

\section{SUMMARY AND CONCLUSION}

In our investigation, we consider a properly chirped laser pulse irradiating to an ultrathin foil and all electrons are pushed out. The chirp of the pulse should be optimized in order to ensure the electrons to be accelerated synchronously. With a proper chirp parameter, the laser driven relativistic electron bunch is confined in nanoscale with high density. Here, the laser intensity is as low as the critical intensity $\left(I=1.35 \times 10^{20} \mathrm{~W} / \mathrm{cm}^{2}\right.$ for $\lambda_{0}=$ $1 \mu \mathrm{m})$. In an actual experiment, a thick reflect foil can be set behind the initial ultrathin foil, which can reflect the laser pulse and extract the DEES.

In conclusion, we study the chirp effect on the laser DEES acceleration regime in the case of laser pulse irradiating an ultrathin foil. Particular attention is paid to the chirp contribution to the confinement control of a DEES. Though the laser pulse is linearly chirped, it is shown the instantaneous frequency does not change monotonously, but increases after a decrease stage. This behavior indicates that an MAS exists during the pulse. Or, in other words, the DEES is strongly accelerated while the experienced fields vary slowly. Based on the analysis of self-field mended chirped laser fields, a nanocontrolled DEES driven by a chirped pulse with critical relativistic intensity is obtained. Therefore the chirped laser pulse offers a reasonable approach to generated DEES for coherent Thomson scattering, with relaxed requirements on the laser intensity.

\section{ACKNOWLEDGMENTS}

This work was supported by NSFC (Grants No. 61108006, No. 11105009, No. 61008016, and No. 11104221) and the Scientific Research Program Funded by Shaanxi Provincial Education Department (Programs No. 11JK0529 and No. 11JK0538). M. W. is also sponsored by SRF for ROCS, SEM.

[1] G. Mourou and T. Tajima, Science 331, 41 (2011).

[2] K. Ta Phuoc, S. Corde, C. Thaury, V. Malka, A. Tafzi, J. P. Goddet, R. C. Shah, S. Sebban, and A. Rousse, Nat. Photonics 6, 308 (2012).

[3] B. Dromey, S. Rykovanov, M. Yeung, R. Hörlein, D. Jung, D. C. Gautier, T. Dzelzainis, D. Kiefer, S. Palaniyppan, R. Shah, J. Schreiber, H. Ruhl, J.C. Fernandez, C. L. S. Lewis, M. Zepf, and B. M. Hegelich, Nat. Phys. 8, 804 (2012).

[4] J. Meyer-ter-Vehn and H. C. Wu, Eur. Phys. J. D 55, 433 (2009).

[5] M. Alterelli and A. Salam, Europhysics News 35, 47 (2004) [http://dx.doi.org/10.1051/epn:2004204].

[6] J. Faure, Y. Glinec, A. Pukhov, S. Kiselev, S. Gordienko, E. Lefebvre, J.-P. Rousseau, F. Burgy, and V. Malka, Nature (London) 431, 541 (2004).

[7] S. P. D. Mangles, C. D. Murphy, Z. Najmudin, A. G. R. Thomas, J. L. Collier, A.E. Dangor, E. J. Divall, P. S. Foster, J. G. Gallacher, C. J. Hooker, D. A. Jaroszynski, A. J. Langley, W. B. Mori, P. A. Norreys, F. S. Tsung, R. Viskup, B.R. Walton, and K. Krushelnick, Nature (London) 431, 535 (2004).

[8] C. G. R. Geddes, C. Toth, J. van Tilborg, E. Esarey, C. B. Schroeder, D. Bruhwiler, C. Nieter, J. Cary, and W. P. Leemans, Nature (London) 431, 538 (2004).

[9] A. Pukhov and J. Meyer-ter-Vehn, Appl. Phys. B 74, 355 (2002).

[10] M. Geissler, J. Schreiber, and J. Meyer-ter-Vehn, New J. Phys. 8, 186 (2006).

[11] H.-C. Wu and J. Meyer-ter-Vehn, Nat. Photonics 6, 304 (2012).

[12] D. Kiefer, A. Henig, D. Jung, D. C. Gautier, K. A. Flippo, S. A. Gaillard, S. Letzring, R. P. Johnson, R. C. Shah, and T. Shimada, J. C. Fernández, V. Kh. Liechtenstein, J. Schreiber, B. M. Hegelich, and D. Habs, Eur. Phys. J. D 55, 427 (2009).

[13] V. V. Kulagin, V. A. Cherepenin, M. S. Hur, and H. Suk, Phys. Rev. Lett. 99, 124801 (2007).

[14] J. Bai, X. Zhong, S. Jiang, Y. Huang, and X. Duan, Nat. Nanotechnol. 5, 190 (2010).

[15] H.-C. Wu and J. Meyer-ter-Vehn, Eur. Phys. J. D 55, 443 (2009).

[16] V. V. Kulagin, V.A. Cherepenin, and H. Suk, Phys. Plasmas 11, 5239 (2004).

[17] P. M. Woodward, J. Inst. Electr. Eng. 93, 1554 (1947).

[18] J. D. Lawson, IEEE Trans. Nucl. Sci. 26, 4217 (1979).

[19] M. Wen, H. C. Wu, L. L. Jin, Y.R. Lu, J.E. Chen, and X. Q. Yan, Phys. Plasmas 19, 083112 (2012). 
[20] D. Strickland and G. Mourou, Opt. Commun. 56, 219 (1985).

[21] J. Faure, J.-R. Marquès, V. Malka, F. Amiranoff, Z. Najmudin, B. Walton, J.-P. Rousseau, S. Ranc, A. Solodov, and P. Mora, Phys. Rev. E 63, 065401 (2001).

[22] R. Hajima and R. Nagai, Phys. Rev. Lett. 91, 024801 (2003).

[23] T. Brabec and F. Krausz, Rev. Mod. Phys. 72, 545 (2000).

[24] D. G. Lee, J.-H. Kim, K.-H. Hong, and C. H. Nam, Phys. Rev. Lett. 87, 243902 (2001).

[25] E.S. Dodd and D. Umstadter, Phys. Plasmas 8, 3531 (2001).

[26] C. B. Schroeder, E. Esarey, C. G. R. Geddes, C. Toth, B. A. Shadwich, J. Van Tilborg, J. Faure, and W. P. Leemans, Phys. Plasmas 10, 2039 (2003).

[27] X. Y. Wu, P.X. Wang, and S. Kawata, Appl. Phys. Lett. 100, 221109 (2012).

[28] F. Sohbatzadeh, S. Mirzanejhad, and M. Ghasemi, Phys. Plasmas 13, 123108 (2006).

[29] A. G. Khachatryan, F. A. van Goor, and K. J. Boller, Phys. Rev. E 70, 067601 (2004).
[30] F. Sohbatzadeh, S. Mirzanejhad, and H. Aku, Phys. Plasmas 16, 023106 (2009).

[31] K. P. Singh, Appl. Phys. Lett. 87, 254102 (2005).

[32] D. N. Gupta and H. Suk, Phys. Plasmas 13, 013105 (2006); 13, 044507 (2006).

[33] J. X. Li, W. P. Zang, and J. G. Tian, Appl. Phys. Lett. 96, 031103 (2010).

[34] B. J. Galow, Y. I. Salamin, T. V. Liseykina, Z. Harman, and C.H. Keitel, Phys. Rev. Lett. 107, 185002 (2011).

[35] Y. I. Salamin, J. X. Li, B. J. Galow, Zoltán Harman, and Christoph H. Keitel, Phys. Rev. A 85, 063831 (2012).

[36] H.-C. Wu, J. Meyer-ter-Vehn, J. Fernández, and B. M. Hegelich, Phys. Rev. Lett. 104, 234801 (2010).

[37] M. Wen, H.-C. Wu, J. Meyer-ter-Vehn, and B. Shen, Eur. Phys. J. D 55, 451 (2009).

[38] C. Nieter and J. R. Cary, J. Comput. Phys. 196, 448 (2004).

[39] L.E. Chipperfield, J.S. Robinson, P.L. Knight, J.P. Marangos, and J.W. G. Tisch, Laser Photonics Rev. 4, 697 (2010). 